\title{
Establishment and evaluation of a new highly metastatic tumor cell line 5a-D-Luc-ZsGreen expressing both luciferase and green fluorescent protein
}

\author{
HITOMI SUDO $^{1}$, ATSUSHI B. TSUII ${ }^{1}$, AYA SUGYO ${ }^{1}$, HIROYUKI TAKUWA ${ }^{2}$, KAZUTO MASAMOTO ${ }^{3}$, \\ YUTAKA TOMITA $^{4}$, NORIHIRO SUZUKI ${ }^{4}$, TAKESHI IMAMURA ${ }^{5}$, MITSURU KOIZUMI $^{6}$ and TSUNEO SAGA ${ }^{1}$ \\ ${ }^{1}$ Diagnostic Imaging Program, Molecular Imaging Center, ${ }^{2}$ Biophysics Program, Molecular Imaging Center, \\ National Institute of Radiological Sciences, Inage-ku, Chiba 263-8555; ${ }^{3}$ Center for Frontier Science and Engineering, \\ University of Electro-Communications, Chofu, Tokyo 182-8585; ${ }^{4}$ Department of Neurology, Keio University \\ School of Medicine, Shinjuku-ku, Tokyo 160-8582; ${ }^{5}$ Department of Molecular Medicine for Pathogenesis, \\ Ehime University Graduate School of Medicine, Ehime 791-0295; ${ }^{6}$ Department of Nuclear Medicine, \\ Cancer Institute Hospital of Japanese Foundation for Cancer Research, Koto-ku, Tokyo 135-8550, Japan
}

Received September 9, 2015; Accepted October 20, 2015

DOI: $10.3892 / \mathrm{ijo} .2015 .3300$

\begin{abstract}
Breast cancer is the most common cancer in women. Although advances in diagnostic imaging for early detection, surgical techniques and chemotherapy have improved overall survival, the prognosis of patients with metastatic breast cancer remains poor. Understanding cancer cell dynamics in the metastatic process is important to develop new therapeutic strategies. Experimental animal models and imaging would be powerful tools for understanding of the molecular events of multistep process of metastasis. In the present study, to develop a new cancer cell line that is applicable to bioluminescence and fluorescence imaging, we transfected the expression vector of a green fluorescent protein ZsGreen1 into a metastatic cell line 5a-D-Luc, which is a subclone of the MDA-MB-231 breast cancer cell line expressing luciferase, and established a new tumor cell line 5a-D-Luc-ZsGreen expressing both luciferase and ZsGreen1. The 5a-D-Luc-ZsGreen cells proliferate more rapidly and have a more invasive phenotype compared with 5a-D-Luc cells following intracardiac injection. Metastasis sites were easily detected in the whole body by bioluminescence imaging and in excised tissues by ex vivo fluorescence imaging. The fluorescence of 5a-D-Luc-ZsGreen cells was not lost after formalin fixation and decalcification. It enabled us to easily evaluate tumor spread and localization at the cellular
\end{abstract}

Correspondence to: Dr Atsushi B. Tsuji, Diagnostic Imaging Program, Molecular Imaging Center, National Institute of Radiological Sciences, 4-9-1 Anagawa, Inage-ku, Chiba 263-8555, Japan E-mail: a_tsuji@nirs.go.jp

Key words: bone metastasis, brain metastasis, animal model, bioluminescence imaging, fluorescence imaging, in vivo imaging, real-time imaging, two-photon excitation microscopy level in microscopic analysis. The strong fluorescence of 5a-DLuc-ZsGreen cells allowed for real-time imaging of circulating tumor cells in cerebral blood vessels of live animals immediately after intracardiac injection of cells using two-photon laser-scanning microscopy. These findings suggest that the 5a-D-Luc-ZsGreen cells would be a useful tool for research on mechanisms of metastatic process in animal models.

\section{Introduction}

Breast cancer is one of the most frequently diagnosed cancers in women (1), and >1.6 million new cases of breast cancer were identified in 2010 over the world (2). The incidence of breast cancer has continued to rise in the last several decades (2). The prognosis of patients with local breast cancer has improved by recent advances in therapy, however, that of patients with distant metastasis is still poor (3). Most patients with metastatic lesions are not treated by surgery since the presence of one metastatic lesion often indicates the presence of widespread systemic disease (3). Chemotherapy, hormonal, and radiation therapies are used for palliative purposes in the metastasis patients, and these therapeutic effects are modest, but achieve statistically significant prolongation of survival (3). If there were more effective therapies for metastasis, the survival rate of patients with metastasis is expected to be further improved. More understanding of the mechanisms of the complicated process of metastasis should help develop more effective therapies. The metastasis process is thought to consist of a serial, interlinked, and selective steps, namely, metastatic cancer cells invade the surrounding stroma, intravasate, survive in the circulation, become arrested in a distant capillary, extravasate, and proliferate in target organ(s) (4). One of the major concepts in metastasis research, the 'seed' and 'soil' theory, was proposed by Stephen Paget in 1889 (5). Paget hypothesized that the interaction between tumor cells (seed) and host environment (soil) determines metastatic outcome (5). This hypothesis 
predicted that the microenvironment of disseminated tumor cells might promote tumor metastasis and contribute to the organ selectivity (3). Experimental animal models would be powerful tools for understanding of the molecular events of multistep process of metastasis. Several previous studies reported that various highly metastatic cells were established and used for elucidating metastatic mechanisms (6-8). In these studies, a luciferase-based bioluminescence imaging technique was used to detect metastatic sites and growth of tumor cells. Bioluminescence imaging has high sensitivity, specificity, and capacity for quantitative analysis. Although this imaging technique is suitable for whole-body imaging, it has poor spatial resolution (1-2 $\mathrm{mm}$ ) due to diffusely scattered photons and results in difficulty in detecting precise localization of tumor cells (9). In contrast, fluorescence imaging has high-resolution and provides localization of tumor cells and molecules associated with metastasis at cellular and subcellular resolutions (10). Thus, a tumor cell expressing both luciferase and fluorescent protein has the potential to be a more powerful tool for elucidation of tumor metastasis mechanisms. We previously established luciferase-expressing MDA-MB-231-5a-D (5a-DLuc), a highly metastatic breast cancer cell line derived from MDA-MB-231 (6). In the present study, we introduced a green fluorescent protein ZsGreen1 to 5a-D-Luc cells, and evaluated the utility for in vivo imaging with several optical imaging methods.

\section{Materials and methods}

Cell culture and transfection. 5a-D-Luc cells, a highly bone metastatic activity cell line derived from MDA-MB-231 (6), were maintained in DMEM medium supplemented with 5\% FBS (JRH Biosciences, Lenexa, KS, USA) in a humidified incubator maintained at $37^{\circ} \mathrm{C}$ with $5 \% \mathrm{CO}_{2}$. The 5a-D-Luc

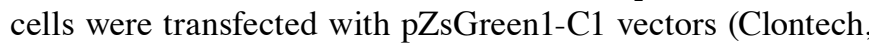
Mountain View, CA, USA) using Lipofectamine 2000 Transfection Reagent (Life Technologies, Carlsbad, CA, USA), and maintained in DMEM with 5\% FBS and $1 \mathrm{mg} / \mathrm{ml} \mathrm{G} 418$ (Roche, Basel, Switzerland) to obtain stable transfectants. We subsequently isolated five subclones of the transfectants stably expressing ZsGreen1 protein (5a-D-Luc-ZsGreen).

In vitro evaluation of $5 a-D-L u c-Z s G r e e n$ cells. Cell proliferation assay was performed using a sulforhodamine B as described previously (11). To evaluate fluorescence and bioluminescence intensities in vitro, 5a-D-Luc-ZsGreen cells were serially diluted from $10^{5}$ to $10^{4}$ cells in 96 -well plates. The fluorescence intensity was measured with a preclinical in vivo imaging system IVIS Lumina (Xenogen, Alameda, CA, USA). Then, the bioluminescence intensity (luciferase activity) was measured using Steady-Glo Luciferase Assay system (Promega, Madison, WI, USA) with the IVIS Lumina.

Animal models. The animal experiments were approved by the Institutional Animal Care and Use Committee of the National Institute of Radiological Sciences, and all animal experiments were conducted in accordance with the institutional guidelines regarding animal care and handling. To evaluate fluorescence and bioluminescence intensities in vivo, female BALB/c-nu/nu mice (5-week-old; CLEA Japan, Tokyo, Japan) were subcutaneously inoculated with a 1:1 mixture of 5a-DLuc-ZsGreen cells $\left(1 \times 10^{3}, 1 \times 10^{4}, 5 \times 10^{4}\right.$ and $1 \times 10^{5}$ cells $)$ and Matrigel (Becton-Dickenson Bioscience, Bedford, MA, USA) on the dorsal side. For intracardiac injection, female nude mice (6-week-old) were anesthetized by inhalation of $3 \%$ isoflurane and injected with 5a-D-Luc or 5a-D-Luc-ZsGreen cells ( $1 \times 10^{5}$ cells) in $100 \mu \mathrm{l}$ PBS into the left cardiac ventricle as described previously (6).

In vivo bioluminescence imaging. Mice were injected with $2.5 \mathrm{mg} / \mathrm{mouse}$ D-luciferin (Wako Pure Chemical Industries, Osaka, Japan) into the tail vain, and the bioluminescence images were acquired with the IVIS Lumina under isoflurane anesthesia (3\%). Mean radiance (photon $/ \mathrm{s} / \mathrm{cm}^{2} / \mathrm{sr}$ ) in region of interest (ROI) drawn over bioluminescence signals was measured using Living Image software (Xenogen).

Ex vivo fluorescence imaging. Brain, bone, and other organs with bioluminescence signals were resected from the mice that received intracardiac injection of 5a-D-Luc-ZsGreen cells at 7, 14, 21 and 28 days after injection. The resected tissues were observed with a fluorescence stereoscopic microscope MZ16F (Leica Microsystems, Wetzlar, Germany).

Two-photon laser-scanning microscopic imaging. For the surgical procedure, a mixture of air, oxygen, and isoflurane $(1.5-2 \%)$ anesthesia was given via face-mask. The head of mouse was fixed with a stereotactic frame, and cranial windows were prepared according to the 'Seylaz-Tomita method' (12). The cranial window was attached over the left side of the somatosensory cortex using dental cement (Luxaflow, DMG, Hamburg, Germany) and centered at $1.8 \mathrm{~mm}$ caudal and $2.5 \mathrm{~mm}$ lateral from the bregma. A custom-made metal plate was affixed to the front of the central skull $(12,13)$. For real-time tracking imaging of tumor cells just after inoculation, mice were anesthetized with $1.5 \%$ isoflurane and was placed supine on a custom-made apparatus. Needle (30 G: Terumo, Tokyo, Japan) with thin plastic tube (catheter) was inserted into left cardiac ventricle of mice and was fixed with dental cement (Ionosit, DMG). The head of a mouse intraperitoneally administered $10 \mathrm{mM}$ SR101 (8 $\mu \mathrm{l} / \mathrm{g}$ body weight) was placed under an objective lens of two-photon laser scanning microscopy (TCS-SP5 MP, Leica Microsystems). Immediately after initiation of two-photon imaging, $1 \times 10^{5}$ of 5a-D-Luc-ZsGreen cells were injected into the mouse through the catheter. The wavelength of excitation laser was $900 \mathrm{~nm}$. An emission signal was separated by a beam splitter $(560 / 10 \mathrm{~nm})$ and simultaneously detected through a band-pass filter for SR101 $(610 / 75 \mathrm{~nm})$ and tumor cells $(525 / 50 \mathrm{~nm})$. A single image plane consisted of 1,024 by 1,024 pixels, and in-plane pixel-size was $0.25-0.45 \mu \mathrm{m}$ depending on an instrumental zoom factor. The images were acquired at depth of $0.2-0.4 \mathrm{~mm}$ from the cortical surface. To observe the moving cancer cells and cerebral vasculature on the surface over the barrel cortex, dynamic imaging was started just after inoculation and conducted at a rate of $0.15 \mathrm{sec}$ per frame for $60 \mathrm{sec}$. For temporal imaging to observe change of tumor cells and blood vessels until day 21 after injection, the mice without a catheter were imaged as described above. 
A

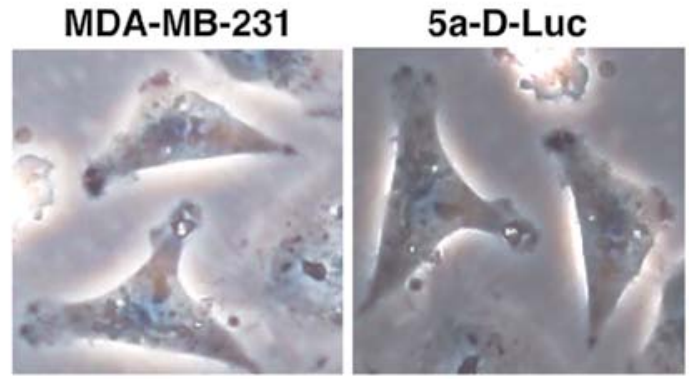

5a-D-Luc-ZsGreen

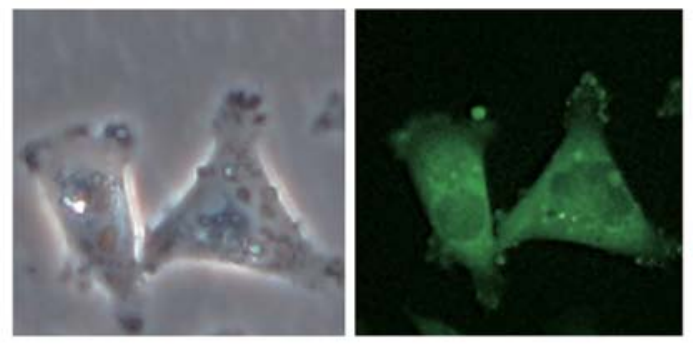

B

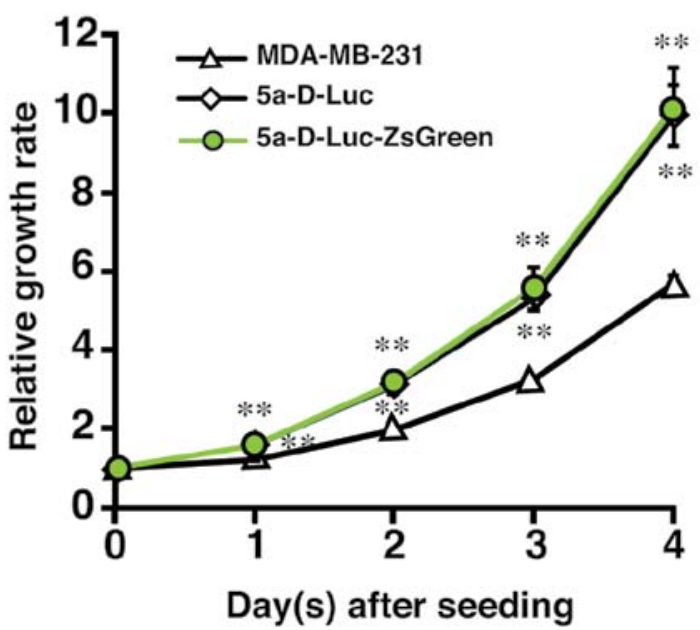

Figure 1. In vitro characterization of 5a-D-Luc-ZsGreen. (A) Representative phase contrast images of MDA-MB-231 and 5a-D-Luc cells (upper panels), and phase contrast and fluorescence images of 5a-D-Luc-ZsGreen cells (lower panels). (B) Cell proliferation of MDA-MB-231, 5a-D-Luc, and 5a-D-Luc-ZsGreen cells. Data represent mean \pm SD from three independent experiments. ${ }^{* *} \mathrm{P}<0.01$ vs. MDA-MB-231.

Histological analysis. After ex vivo imaging, the bone tissues were fixed in $10 \%$ neutral-buffered formalin, decalcified with EDTA treatment and embedded in paraffin. The tissue sections (1- $\mu \mathrm{m}$ thick) were deparaffinized and stained with H\&E or tartrate resistant acid phosphatase (TRAP) staining kit (Wako Pure Chemical Industries). After TRAP staining, the sections were counterstained with hematoxylin. The brains were resected, embedded in frozen section compound (Leica Microsystems) and frozen for cryostat sectioning $(8-\mu \mathrm{m}$ thick). Immunofluorescence staining was conducted using rat anti-CD31 antibody (BD Pharmingen, San Diego, CA, USA) and Alexa Fluor 594-labeled anti-rat IgG (Molecular Probes, Eugene, OR, USA) or rabbit anti-claudin-5 antibody (Abcam, Cambridge, UK) and Alexa Fluor 594-labeled anti-rabbit IgG (Molecular Probes). The slides were mounted in Vectashield mounting medium with DAPI (Vector Laboratories, Burlingame, CA, USA). The stained tissues were observed with a fluorescence microscope BX53 (Olympus, Tokyo, Japan).

Statistical analysis. Statistical differences was analyzed by ANOVA with the Dunnett's multiple comparison test, or by Student's t-test. Correlation between the number of cells, and bioluminescence or fluorescence intensity was calculated by Pearson's product correlation coefficient.

\section{Results}

5a-D-Luc-ZsGreen cells. We transfected the ZsGreen1-expressing vector into 5a-D-Luc cells derived from MDA-MB-231 $(6,7)$, selected stably ZsGreen1-expressing cells, and then isolated five subclones. One of the five subclones that showed the strongest fluorescence intensity was selected for the following experiments and was named 5a-D-Luc-ZsGreen. In the 5a-D-Luc-ZsGreen cells, the signal of green fluorescence was observed in the cytoplasm (Fig. 1A) and the fluorescence intensity seemed to be stably maintained $\geq 12$ passages in vitro (data not shown). There were no morphological differences between MDA-MB-231, 5a-D-Luc, and 5a-D-Luc-ZsGreen cells (Fig. 1A). The 5a-D-Luc and 5a-D-Luc-ZsGreen cells proliferated more rapidly than the parental MDA-MB-231 cells $(\mathrm{P}<0.01)$ (Fig. 1B). No significant difference in growth rate was observed between 5a-D-Luc and 5a-D-Luc-ZsGreen cells (Fig. 1B).

In vitro evaluation of $5 a-D-L u c-Z s G r e e n$. The bioluminescence and fluorescence intensities of 5a-D-Luc-ZsGreen were measured in vitro. Both bioluminescence and fluorescence intensities were detected when $>10,000$ cells were used. The number of cells was significantly correlated with bioluminescence $\left(R^{2}=0.92, P<0.01\right)$ and fluorescence intensity $\left(R^{2}=0.99\right.$, $\mathrm{P}<0.01$ ) (Fig. 2A and B).

In vivo evaluation of $5 a-D$-Luc-ZsGreen. To evaluate the correlation of the number of cells with the bioluminescence and fluorescence intensities in vivo, $3 \mathrm{~h}$ after subcutaneous inoculation of 5a-D-Luc-ZsGreen cells into nude mice, the bioluminescence and fluorescence intensities were measured. Both bioluminescence and fluorescence intensities were detected when $\geq 10,000$ cells were injected (Fig. 2C and E). There were significant correlations between the number of cells, and bioluminescence $\left(\mathrm{R}^{2}=0.89, \mathrm{P}<0.01\right)$ or fluorescence intensity $\left(\mathrm{R}^{2}=0.84, \mathrm{P}<0.01\right)($ Fig. $2 \mathrm{D}$ and $\mathrm{F})$.

Comparison of metastatic ability of 5a-D-Luc-ZsGreen and $5 a-D-L u c$. To compare the metastatic ability between 5a-D-Luc and 5a-D-Luc-ZsGreen, these cells were injected into the left cardiac ventricle. As shown in Fig. 3A, bioluminescence signals were detected at 7 days after inoculation with $1 \times 10^{5}$ cells, and the signal intensity and the number of metastatic sites increased over time. The signal intensity in metastatic regions in 5a-D-Luc-ZsGreen-inoculated mice became stronger than that in 5a-D-Luc-inoculated mice 21 and 28 days after inoculation $(\mathrm{P}<0.01)$ (Fig. 3B). According to ex vivo imaging and histological analysis, the 5a-D-Luc- 
A
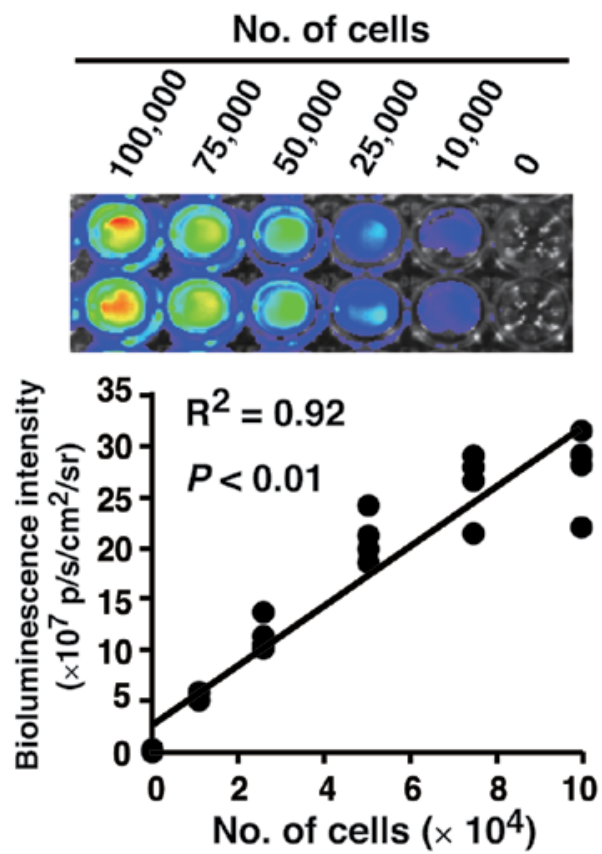

C

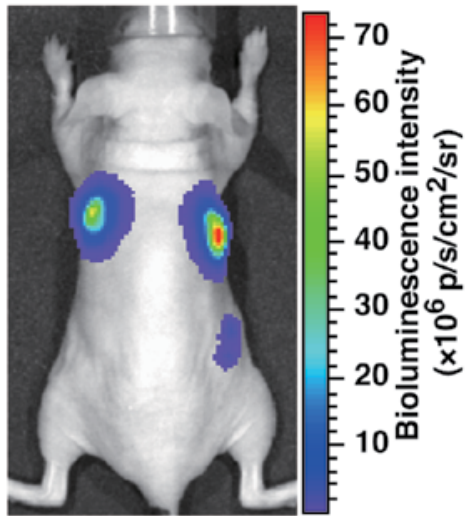

E

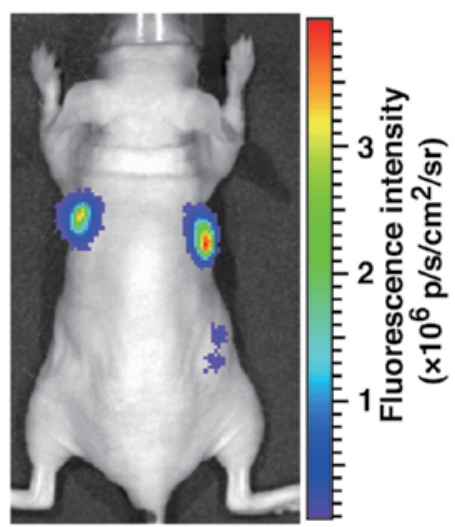

B
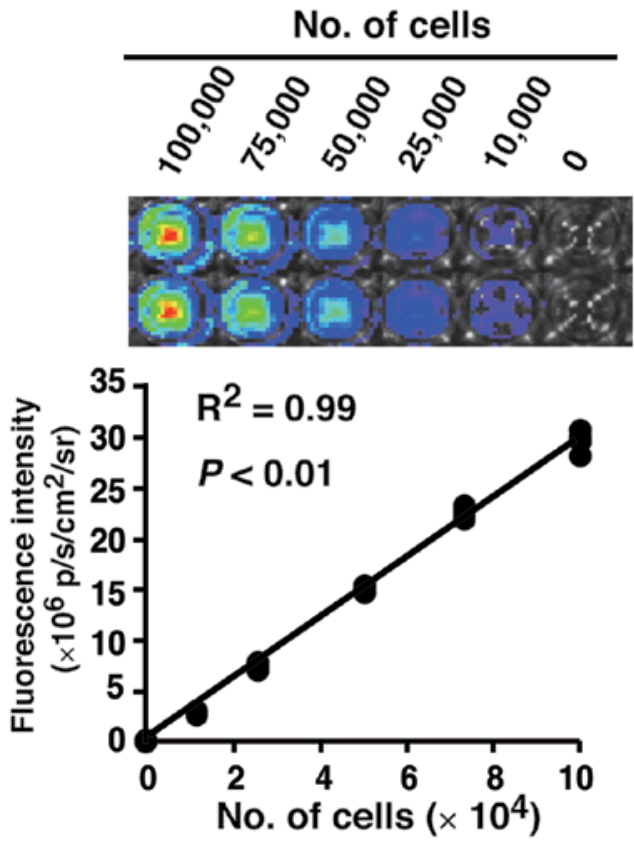

D

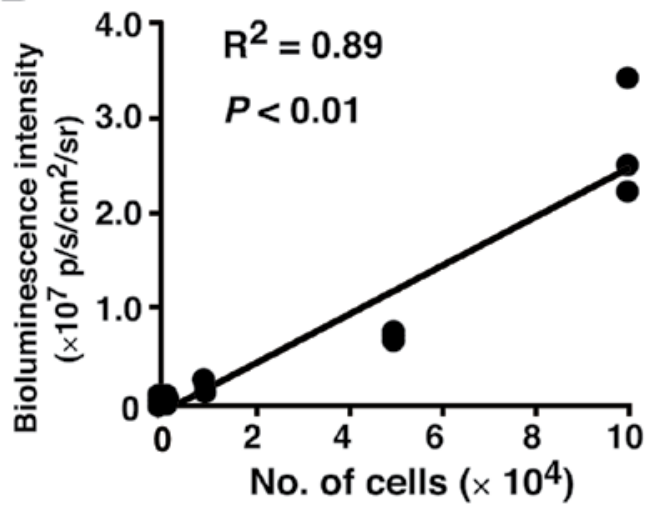

$\mathbf{F}$

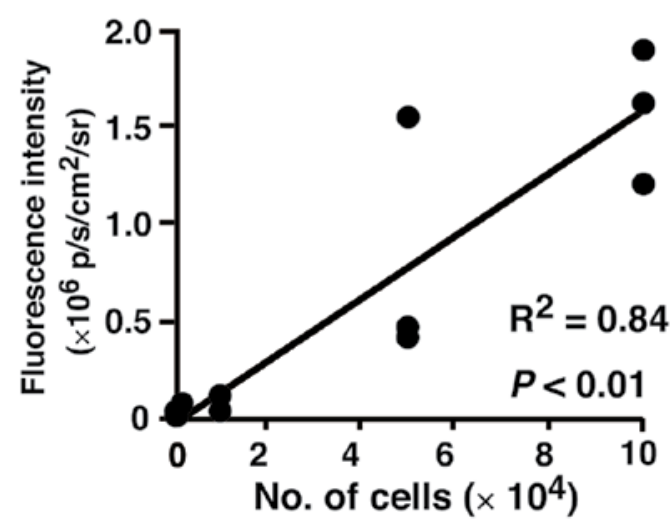

Figure 2. Quantification of luminescence and fluorescence intensities in 5a-D-Luc-ZsGreen cells. (A) Bioluminescence image of cells and correlation analysis of bioluminescence intensity with the number of cells in vitro. (B) Fluorescence image of cells and correlation analysis of fluorescence intensity with the number of cells in vitro. (C) Representative bioluminescence images of mice subcutaneously inoculated with cells. Upper right, $1 \times 10^{5}$; upper left, $5 \times 10^{4}$; lower right, $1 \times 10^{4}$; lower left, $1 \times 10^{3}$ cells in the mouse. (D) Correlation analysis between bioluminescence intensity and the number of cells (C). (E) Representative fluorescence image of mice subcutaneously inoculated with cells. Upper right, $1 \times 10^{5}$; upper left, $5 \times 10^{4}$; lower right, $1 \times 10^{4}$; lower left, $1 \times 10^{3}$ cells in the mouse. (F) Correlation analysis between fluorescence intensity and the number of cells (E).

ZsGreen cells metastasized to bone (all of five mice), brain (all of five mice), adrenal gland (two of five mice), and lymph node (one of five mice) by day 28 after intracardiac inoculation. The 5a-D-Luc cells metastasized to bone (all of five mice) 

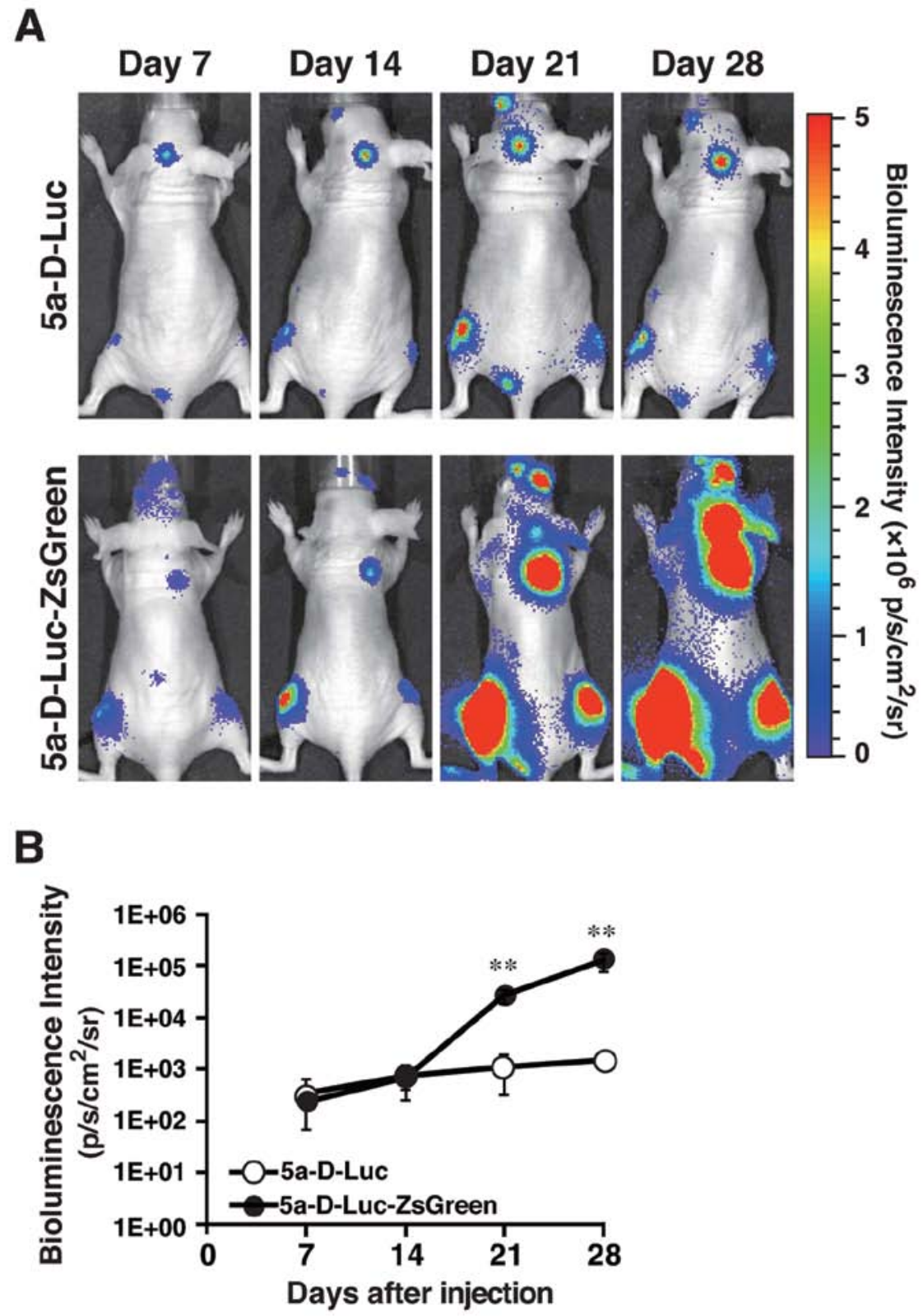

Figure 3. In vivo bioluminescence images of mice administered intracardiac injection of 5a-D-Luc and 5a-D-Luc-ZsGreen cells. (A) Representative bioluminescence images of the models of intracardiac injection of 5aD-Luc or 5a-D-Luc-ZsGreen cells. (B) The bioluminescence intensity of metastatic sites in mice. Data represent mean $\pm \mathrm{SD}$ from five mice. ${ }^{* *} \mathrm{P}<0.01$, vs. 5a-D-Luc.

and brain (two of five mice), other tissue metastasis was not observed by day 28 after intracardiac inoculation.

Bone metastasis of 5a-D-Luc-ZsGreen cells. In all of the 5a-D-Luc-ZsGreen-injected mice, bone metastases in femora, tibiae, and spine were observed. Other bone metastases were also observed in mandibles (two of five mice), scapula (three of five mice), wrist joint (three of five mice), and incisor (two of five mice). The ZsGreen1 fluorescence was clearly detected in the metastasized regions by ex vivo imaging by fluorescence stereomicroscopy (Fig. 4A). The ZsGreen1 fluorescence was detected in the sections of metastasized bone after formalin fixation and decalcification (Fig. 4B and C). By the histological analysis of bone metastasis sites, bone marrow cavity was almost completely replaced by metastatic tumor cells at day 28 after intracardiac injection (Fig. 4B). Fluorescence imaging and TRAP staining showed clearly many osteoclasts were localized between the tumor cells and bone (Fig. 4C).

Multifocal metastasis of 5a-D-Luc-ZsGreen cells in the brain. As shown in Fig. 5A, the green fluorescence of the tumor cells was widely distributed in the whole brain just after intracardiac injection. At 7 days after injection, only small and weak fluorescence foci were detected, and subsequently the intensity and size of metastatic foci increased over time (Fig. 5A). These findings were consistent with the results in bioluminescence imaging as shown in Fig. 3. The 5a-D-Luc-ZsGreen cells developed multifocal metastasis in the cerebrum, the cerebellum, and leptomeninges. This feature is common to brain metastasis in breast cancer patients (14). 

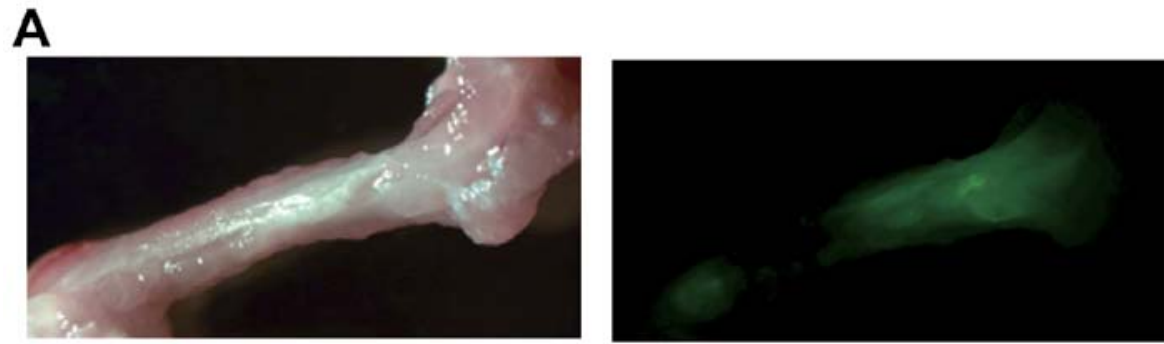

\section{B}
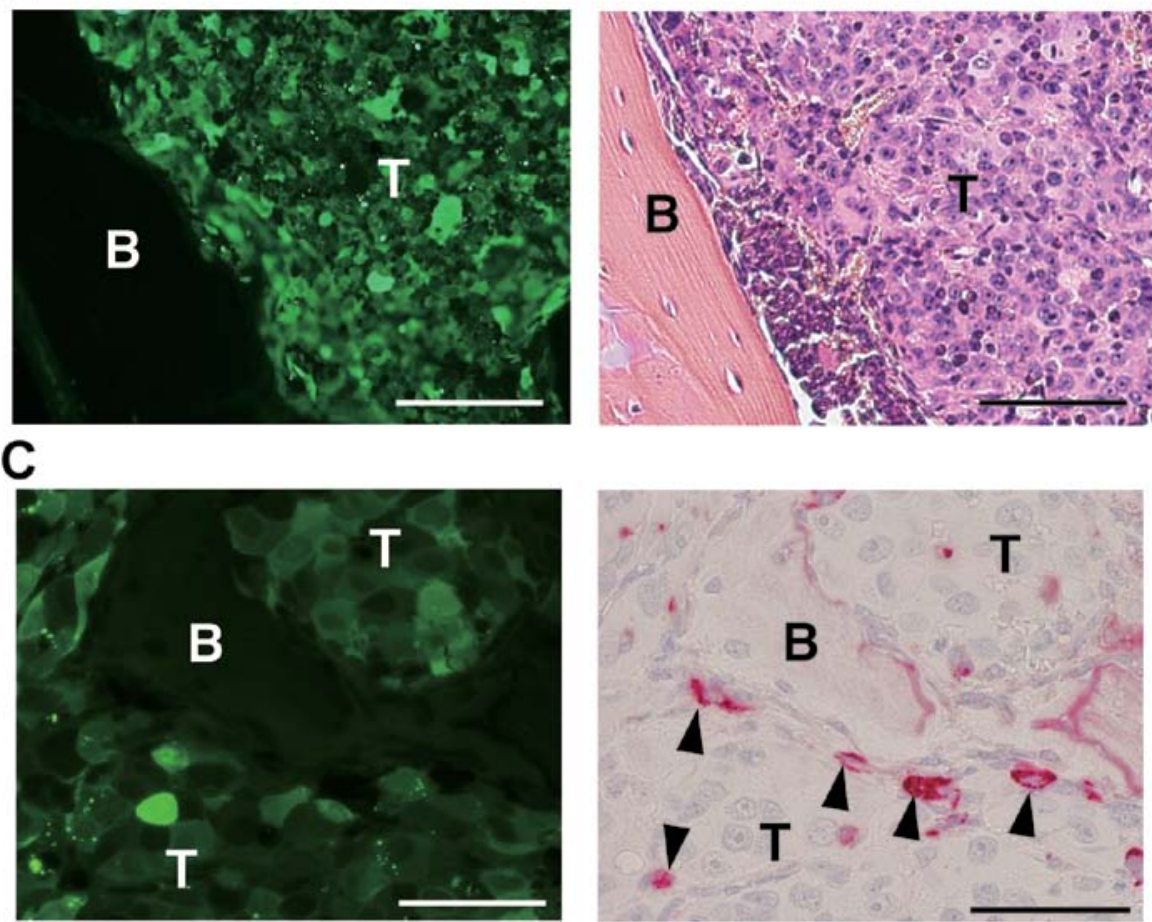

Figure 4. Bone metastasis of 5a-D-Luc-ZsGreen cells. (A) Representative ex vivo images of bone metastasis (left panel, bright field; right panel, fluorescence) (B) Representative fluorescence (left) and H\&E staining (right) images in the same section of a femur from a mouse 28 days after intracardiac injection with 5a-D-Luc-ZsGreen cells. T, tumor; B, bone; black or white bars, $100 \mu \mathrm{m}$. (C) Representative fluorescence (left panel) and TRAP staining (right panel) images in the same section of a femur from a mouse 28 days after intracardiac injection with 5a-D-Luc-ZsGreen cells. Arrowheads represent osteoclasts. T, tumor; $\mathrm{B}$, bone; black or white bars, $50 \mu \mathrm{m}$.

Immunofluorescence staining of CD31 showed a close association of vessels with tumor cells. At $5 \mathrm{~min}$ after injection, tumor cells were detected within brain microvessels, especially at vascular branching points (Fig. 5B, upper left panel). At day 14 , tumor cells were extravasated into surrounding tissues, and no cell was observed within the blood vessels (Fig. 5B, upper right panel). At day 21, tumor cells proliferated along microvessels (Fig. 5B, lower left panel), and alterations of vessel morphology and density were also observed at day 28 (Fig. 5B, lower right panel). By immunostaining for claudin-5, a tight junction marker used for evaluating the integrity of blood-brain barrier (BBB), the lack of claudin-5 expression was noted in a part of microvessels near tumor metastatic sites at day 28 (Fig. 5C).

Real-time tracking and temporal imaging of 5a-D-LucZsGreen cells in the brain. Finally, in vivo two-photon laser-scanning microscopy allowed real-time tracking of circulating tumor cells in bloodstream of the brain. The tumor cells traveled through the bloodstream in brain vessels just after intracardiac injection (Fig. 5D). At $20 \mathrm{sec}$ after injection, some tumor cells were plugged in a blood vessel branching point (Fig. 5D), and most of these cells disappeared by day 5 after injection (data not shown). Temporal imaging using two-photon microscopy revealed that: i) tumor cells proliferated in the brain parenchyma and microvessels formed in a single direction, and ii) microvessel density increased as tumor cells proliferated until day 21 after intracardiac injection (Fig. 5E).

\section{Discussion}

In the present study, we transfected an expressing vector of ZsGreen1 into a metastatic cell line 5a-D-Luc $(6,7)$ which is a subclone of the MDA-MB-231 breast cancer cell line expressing luciferase, and successfully established a new tumor cell line 5a-D-Luc-ZsGreen expressing both luciferase and ZsGreen1. Our in vivo experiments showed that the metastatic sites of 5a-D-Luc-ZsGreen and 5a-D-Luc cells were detected by bioluminescence imaging from at least day 7 after intracardiac injection. The 5a-D-Luc-ZsGreen cells developed multiple metastases at high frequencies in bones and the brain 
A

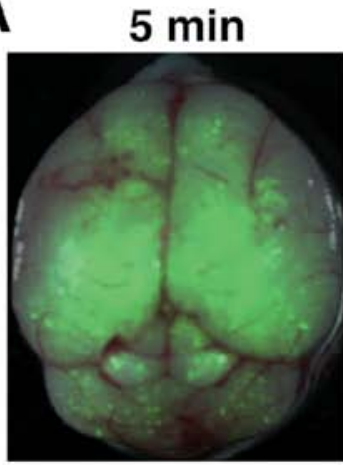

B
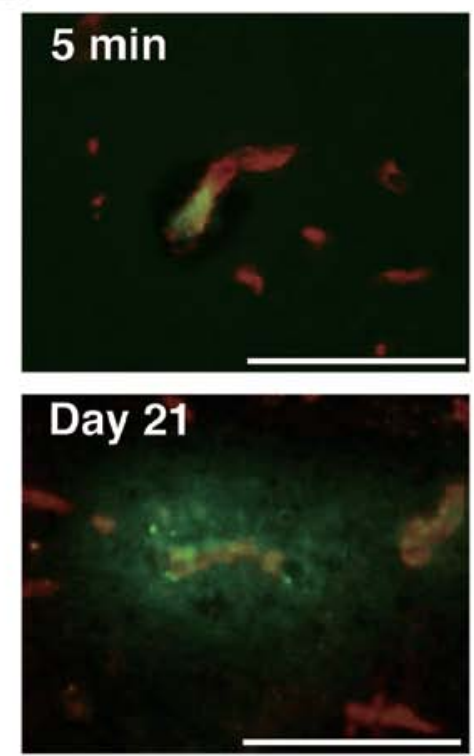

C

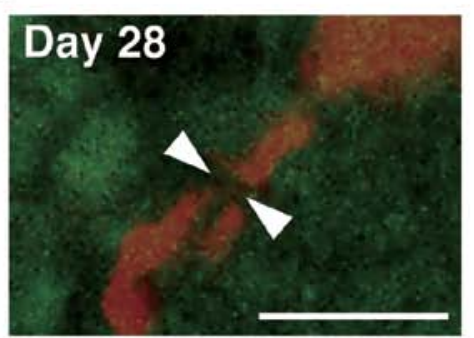

Day 7
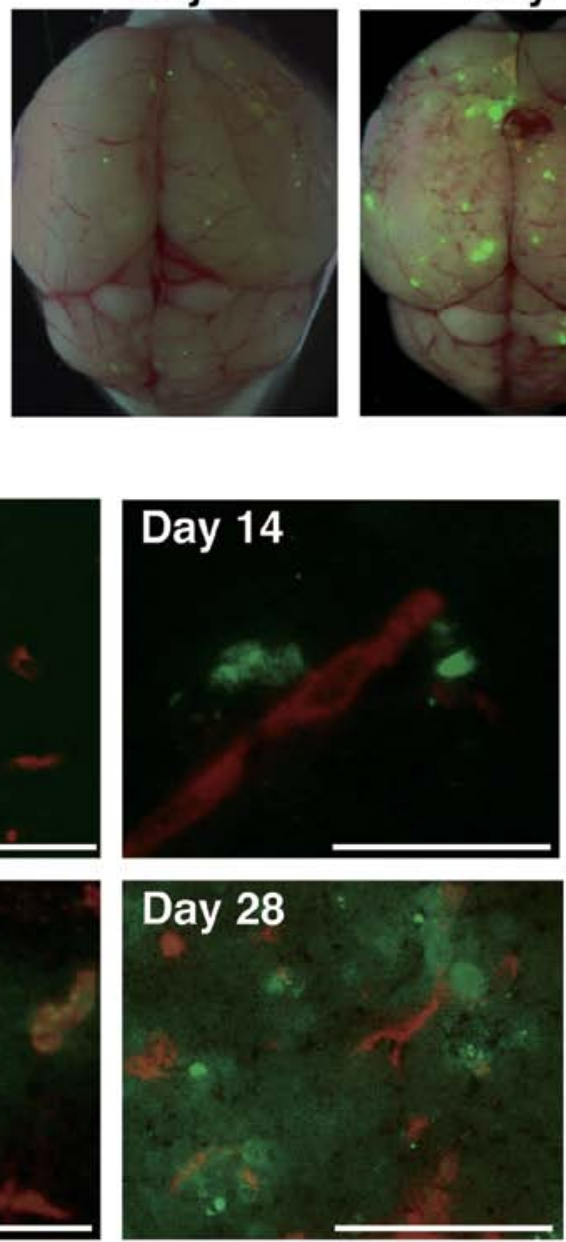

E

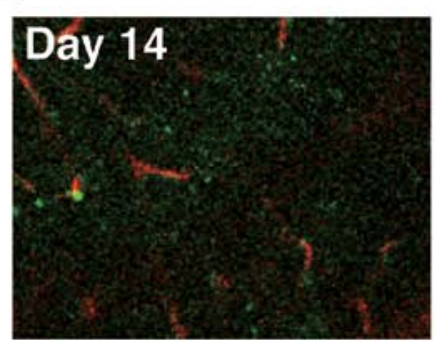

Day 14

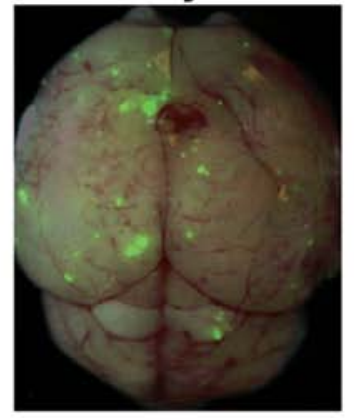

D
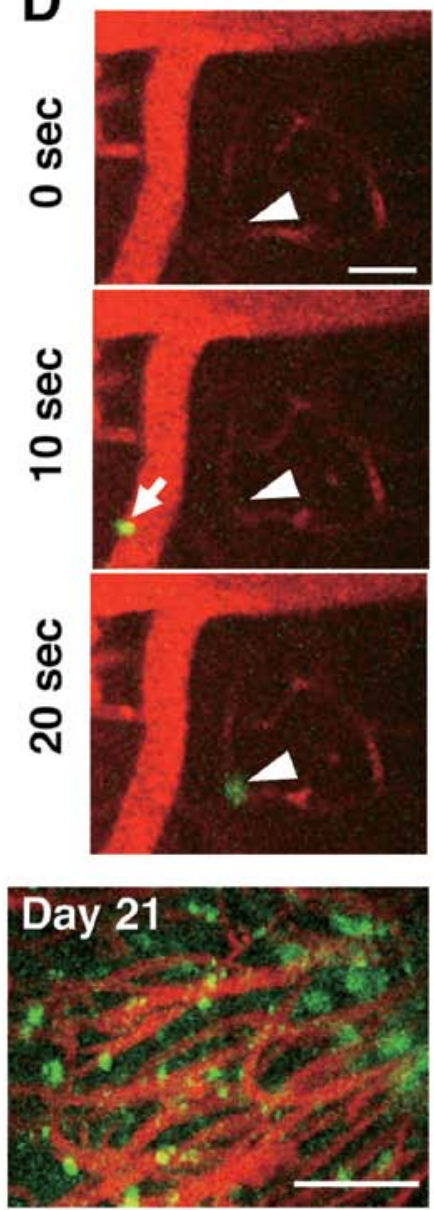

Figure 5. Brain metastasis of 5a-D-Luc-ZsGreen cells. (A) Representative ex vivo fluorescence images of the brain after intracardiac injection with 5a-DLuc-ZsGreen cells. (B) Immunofluorescence staining for CD31 (red) 5 min, and 14, 21, and 28 days after intracardiac injection with 5a-D-Luc-ZsGreen cells (green). Bar, $100 \mu \mathrm{m}$. (C) Immunofluorescence staining for claudin-5 (red) at 28 days after intracardiac injection with 5a-D-Luc-ZsGreen cells (green). Arrowheads represent lack of claudin-5 expression. Bar, $50 \mu \mathrm{m}$. (D) Temporal images immediately after intracardiac injection with 5a-D-Luc-ZsGreen cells (green) using two-photon microscopy. Arrowheads represent the blood vessel branch point where tumor cells were arrested 20 sec after injection. Arrow represents tumor cells traveled through the bloodstream. Bar, $100 \mu \mathrm{m}$. (E) Representative images of the blood vessels and tumor cells at day 14 (left panel) and at day 21 (right panel) after injection using two-photon microscopy. Bar, $100 \mu \mathrm{m}$.

like the parental cell line 5a-D-Luc. Unexpectedly, the signal intensity of bioluminescence in 5a-D-Luc-ZsGreen tumors was significantly stronger than that of 5a-D-Luc tumors at 21 and 28 days after injection, even though in vitro cell growth of the two lines was not different. The bioluminescence intensity of 5a-D-Luc-ZsGreen and 5a-D-Luc cells was linearly correlated with the number of cells in vitro and in vivo. These results suggest that the 5a-D-Luc-ZsGreen cells have a higher growth rate of tumor in vivo compared with 5a-D-Luc cells.
Interestingly, 5a-D-Luc-ZsGreen cells metastasized not only to femur and the brain but also to incisor, adrenal gland, and lymph node, unlike 5a-D-Luc cells. These findings suggest that 5a-D-Luc-ZsGreen cells acquired a more invasive and highly metastatic phenotype compared with 5a-D-Luc cells. Further comprehensive characterization of the two lines may provide new insight into the molecular mechanisms of metastasis.

The approximate sites of metastatic 5a-D-Luc-ZsGreen tumors were easily detected in live animal by bioluminescence 
imaging, while the precise sites in excised organs and tissues were easily detected by fluorescence stereoscopic microscopy. It allows us to do efficient sampling of appropriate tissues for further pathologic analysis. In tissue sections, the strong fluorescence of 5a-D-Luc-ZsGreen cells enables us to easily evaluate tumor spread and localization at the cellular level. Wang et al established MDA-MB-231 cells expressing both eGFP and luciferase for an orthotopic mammary tumor model, and assessed the expression of eGFP and luciferase in vitro and in vivo (15). Those cells were used only for frozen sections (15), while the 5a-D-Luc-ZsGreen cells could be used not only for frozen sections but also decalcified and paraffin-embedded sections in the present study because the fluorescence intensity of ZsGreen1 remains after paraffin fixation and decalcification (16). In addition, the ZsGreen1 protein provides higher fluorescence intensities compared with eGFP (17), suggesting that 5a-D-Luc-ZsGreen cells would be detected more easily in vitro and in vivo. The above suggests that 5a-D-Luc-ZsGreen cells could be applied to all kinds of histological techniques and useful for research on the interaction of tumor cells with osteoclasts and the influence of tumor cells on BBB integrity at cellular level in vivo.

Our results showed that two-photon laser-scanning microscopy enabled real-time tracking and temporal imaging of 5a-D-Luc-ZsGreen cells at single cell level in vivo. In our previous study, we showed that two-photon laser-scanning microscopy allowed for three-dimensional volume imaging of rhodamine-labeled cerebral microvessels up to a depth of $800 \mu \mathrm{m}$ in the cortex of anesthetized mice (18). In the present study, 5a-D-Luc-ZsGreen cells were successfully detected up to a depth of $600 \mu \mathrm{m}$ over 21 days after intracardiac injection. Moreover, we succeeded in temporal imaging of increased angiogenesis accompanied by tumor growth at metastatic sites. The results confirmed the stable and bright fluorescence of 5a-D-Luc-ZsGreen cells in vivo. The bright fluorescence was also supported by our results of real-time tracking of circulating 5a-D-Luc-ZsGreen cells in the brain tissue microcirculation. Taken together, two-photon laserscanning microscopy in 5a-D-Luc-ZsGreen cells enabled us to determine the detailed behavior of tumor cells during the process of metastasis. Future studies should focus on each process of tumor cell adhesion to microvessels, extravasation, colonization, and influences on brain parenchymal cells, and these analyses may provide new insight into the relationship of circulating tumor cells with blood flow and vascular walls.

In conclusion, 5a-D-Luc-ZsGreen cells allowed for easy detection of metastatic sites in the whole body and detailed histological evaluation of the relationship between tumor cells and molecules involved in metastasis at single cell level. Moreover, the strong fluorescence of the cells enabled realtime tracking imaging of circulating tumor cells immediately after intracardiac injection and temporal imaging of tumor proliferation and angiogenesis in vivo. These data demonstrated that our cells would be a useful and powerful tool to further understanding the mechanisms of tumor invasion, such as extravasation and infiltration to tissue, at single cell level. Interestingly, 5a-D-Luc-ZsGreen cells are more metastatic compared with the parental 5a-D-Luc cells, suggesting that further comprehensive characterization of the two lines may provide a new insight into mechanisms of metastasis.

\section{Acknowledgements}

The authors thank Yuriko Ogawa for technical assistance and the staff in the Laboratory Animal Sciences section for animal management.

\section{References}

1. Ferlay J, Shin HR, Bray F, Forman D, Mathers C and Parkin DM: Estimates of worldwide burden of cancer in 2008: GLOBOCAN 2008. Int J Cancer 127: 2893-2917, 2010.

2. Forouzanfar MH, Foreman KJ, Delossantos AM, Lozano R, Lopez AD, Murray CJ and Naghavi M: Breast and cervical cancer in 187 countries between 1980 and 2010: A systematic analysis. Lancet 378: 1461-1484, 2011.

3. Steeg PS: Tumor metastasis: Mechanistic insights and clinical challenges. Nat Med 12: 895-904, 2006.

4. Talmadge JE and Fidler IJ: AACR centennial series: the biology of cancer metastasis: historical perspective. Cancer Res 70: 5649-5669, 2010

5. Paget S: The distribution of secondary growths in cancer of the breast. 1889. Cancer Metastasis Rev 8: 98-101, 1989.

6. Ehata S, Hanyu A, Fujime M, Katsuno Y, Fukunaga E, Goto K, Ishikawa Y, Nomura K, Yokoo H, Shimizu T, et al: Ki26894, a novel transforming growth factor-beta type I receptor kinase inhibitor, inhibits in vitro invasion and in vivo bone metastasis of a human breast cancer cell line. Cancer Sci 98: 127-133, 2007.

7. Katsuno Y, Hanyu A, Kanda H, Ishikawa Y, Akiyama F, Iwase T, Ogata E, Ehata S, Miyazono K and Imamura T: Bone morphogenetic protein signaling enhances invasion and bone metastasis of breast cancer cells through Smad pathway. Oncogene 27: 6322-6333, 2008.

8. Bos PD, Zhang XH, Nadal C, Shu W, Gomis RR, Nguyen DX, Minn AJ, van de Vijver MJ, Gerald WL, Foekens JA, et al: Genes that mediate breast cancer metastasis to the brain. Nature 459: 1005-1009, 2009.

9. Servais EL, Colovos C, Bograd AJ, White J, Sadelain M and Adusumilli PS: Animal models and molecular imaging tools to investigate lymph node metastases. J Mol Med Berl 89: 753-769, 2011.

10. Kain SR, Adams M, Kondepudi A, Yang TT, Ward WW and Kitts P: Green fluorescent protein as a reporter of gene expression and protein localization. Biotechniques 19: 650-655, 1995.

11. Sudo H, Tsuji AB, Sugyo A, Ogawa Y, Sagara M and Saga T: ZDHHC8 knockdown enhances radiosensitivity and suppresses tumor growth in a mesothelioma mouse model. Cancer Sci 103: 203-209, 2012.

12. Tomita Y, Kubis N, Calando Y, Tran Dinh A, Méric P, Seylaz J and Pinard E: Long-term in vivo investigation of mouse cerebral microcirculation by fluorescence confocal microscopy in the area of focal ischemia. J Cereb Blood Flow Metab 25: 858-867, 2005.

13. Takuwa H, Masamoto K, Yamazaki K, Kawaguchi H, Ikoma Y, Tajima Y, Obata T, Tomita Y, Suzuki N, Kanno I, et al: Long-term adaptation of cerebral hemodynamic response to somatosensory stimulation during chronic hypoxia in awake mice. J Cereb Blood Flow Metab 33: 774-779, 2013.

14. Weil RJ, Palmieri DC, Bronder JL, Stark AM and Steeg PS: Breast cancer metastasis to the central nervous system. Am J Pathol 167: 913-920, 2005.

15. Wang K, Xie S, Ren Y, Xia H, Zhang X and He J: Establishment of a bioluminescent MDA-MB-231 cell line for human triplenegative breast cancer research. Oncol Rep 27: 1981-1989, 2012.

16. Harrell JC, Dye WW, Allred DC, Jedlicka P, Spoelstra NS, Sartorius CA and Horwitz KB: Estrogen receptor positive breast cancer metastasis: Altered hormonal sensitivity and tumor aggressiveness in lymphatic vessels and lymph nodes. Cancer Res 66: 9308-9315, 2006.

17. Bell P, Vandenberghe LH, Wu D, Johnston J, Limberis $\mathrm{M}$ and Wilson JM: A comparative analysis of novel fluorescent proteins as reporters for gene transfer studies. J Histochem Cytochem 55: 931-939, 2007.

18. Masamoto K, Tomita Y, Toriumi H, Aoki I, Unekawa M, Takuwa H, Itoh Y, Suzuki N and Kanno I: Repeated longitudinal in vivo imaging of neuro-glio-vascular unit at the peripheral boundary of ischemia in mouse cerebral cortex. Neuroscience 212: 190-200, 2012. 